\title{
Observer Design for a Class of Exothermal Plug-Flow Tubular Reactors
}

\author{
N. Barje*, M.E. Achhab, V. Wertz \\ LINMA, Department of Mathematics, Faculty of Sciences, Universit Chouaib Doukkali, Morocco \\ LINMA, Department of Mathematics, Faculty of Sciences, Universit Chouaib Doukkali, Morocco \\ CESAME, Universit Cathoulique de Louvain, Louvain-La-Neuve, Belgium \\ *Corresponding author E-mail: nbarje@yahoo.fr
}

\begin{abstract}
A semi-linear reduced-order state estimator is presented to reconstruct approximately the state variable initially unknown of a class of nonlinear tubular reactors models, namely the exothermal plug-flow tubular reactor involving sequential reactions for which the kinetics depends on both the temperature and the reactant concentration. Our conception is based on bounded observations and the analysis of the nonlinear set of partial differential equations. It is shown that the given observer design admits a global unique solution and ensures asymptotic state estimator with exponentially decay error, when only the temperature is available for measurement at the reactor outlet. Simulation results are also presented showing the effectiveness of the proposed observer design.
\end{abstract}

Keywords: dissipativity, tubular reactor, nonlinear distributed parameter systems, $C_{0}-$ semigroup.

\section{Introduction}

An intensive research activity has been dedicated to the study of the observability and controllability of (bio)chemical process in the last decades (see [1], [2], [3], [4], [5], [6] and all the references within). This interest can be explained by the potential of these process to improve the productivity. In such systems, the states, inputs, and outputs depend on a spatial variable. This dependance, along with additional aspects such as the boundary conditions and nonlinearities caused by the kinetics of the reactants involved in the process, increase the complexity of the state estimation problem and of the design methods.

For system analysis as well as for control design problems, many surveys has been dedicated to a large class of partial differential equations in linear models. However, an important number of questions remained unsolved so far in the case of nonlinear models. In particular, for the state control of the nonlinear tubular reactor model, the state must be estimated using state estimators (observers).

In this direction, this paper investigates the question of the conception of a exponential reduced-order observer for a class of a chemical non-isothermal tubular reactor for which kinetics is characterized by first-order kinetics with respect to the reactant concentration $C(\mathrm{~mol} / \mathrm{l})$ and by an Arrhenius-type dependence with respect to the temperature $T(K)$, when only the measurements of the temperature may occur at the reactor output. The dynamics of the process are described by the following two energy and mass balance partial differential equations (PDEs) (see $[2])$ :

$$
\begin{aligned}
& \frac{\partial T}{\partial \tau}=-v \frac{\partial T}{\partial \zeta}-\frac{4 h}{\rho C_{p} d}\left(T-T_{c}\right)-\frac{\Delta H}{\rho C_{p}} k_{0} C e^{\frac{-E}{R T}} \\
& \frac{\partial C}{\partial \tau}=-v \frac{\partial C}{\partial \zeta}-k_{0} C e^{\frac{-E}{R T}}
\end{aligned}
$$

where the boundary conditions are given, for $\tau \geq 0$, by:

$$
T(0, \tau)=T_{i n}, C(0, \tau)=C_{\text {in }}
$$


and the initial conditions are given, for $0 \leq \zeta \leq L$, by:

$$
T(\zeta, 0)=T_{0}(\zeta), C(\zeta, 0)=C_{0}(\zeta)
$$

In the equations above, the following parameters $v, \Delta H, \rho, C_{p}, k_{0}, E, R, h, d, T_{c}$ hold for the superficial fluid velocity, the heat of reaction, the density, the specific heat, the kinetic constant, the activation energy, the ideal gas constant, the wall heat transfer coefficient, the reactor diameter, the coolant temperature. $T_{i n}$ and $C_{i n}$ are respectively the inlet temperature and the inlet reactant concentration. $\tau, \zeta$ and $L$ denote the time and space independent variables, and the length of the reactor, respectively. Finally $T_{0}$ and $C_{0}$ denote the initial temperature and reactant concentration profiles.

From a physical point of view it is expected that for all $(z, t) \in[0,1] \times[0,+\infty)$,

$$
0 \leq T(z, t) \leq T_{\max } \text { and } 0 \leq C(z, t) \leq C_{i n}
$$

where $T_{\max }$ could possibly be equal to $+\infty$. Let consider the following dimensionless state transformation:

$$
x_{1}=\frac{T-T_{i n}}{T_{i n}}, x_{c}=\frac{T_{c}-T_{i n}}{T_{i n}}, x_{2}=\frac{C_{i n}-C}{C_{i n}},
$$

Let us consider also dimensionless time $t$ and space $z$ variables:

$$
t=\frac{\tau v}{L}, z=\frac{\zeta}{L} \text {. }
$$

We shall assume in the rest of the paper that the coolant temperature $T_{c}$ is equal to the inlet temperature $T_{i n}$ ( i.e $x_{c} \equiv 0$ ), since $x_{c}$ will be eliminated in the equation of the reconstruction error between the plan state and the observer state.

Then we obtain the following equivalent representation of the model (1)-(4):

$$
\begin{array}{cc}
\frac{\partial x_{1}}{\partial t}= & -\frac{\partial x_{1}}{\partial z}-\beta x_{1}+\alpha \delta\left(1-x_{2}\right) \exp \left(\frac{\mu x_{1}}{1+x_{1}}\right) \\
\frac{\partial x_{2}}{\partial t}= & -\frac{\partial x_{2}}{\partial z}+\alpha\left(1-x_{2}\right) \exp \left(\frac{\mu x_{1}}{1+x_{1}}\right)
\end{array}
$$

with the boundary conditions:

$$
x_{1}(z=0, t)=0, \quad x_{2}(z=0, t)=0
$$

and the initial conditions:

$$
x_{1}(z, t=0)=x_{1}^{0}, \quad x_{2}(z, t=0)=x_{2}^{0}
$$

The parameters $\alpha, \beta, \delta$ and $\mu$ are related to the original parameters as follows:

$$
\mu=\frac{E}{R T_{i n}}, \quad \alpha=\frac{k_{0} L}{v} \exp (-\mu), \quad \beta=\frac{4 h L}{\rho C_{p} d v}, \quad \delta=-\frac{\Delta H}{\rho C_{p}} \frac{C_{i n}}{T_{i n}} .
$$

The real constants $\alpha, \beta$ and $\mu$ are strictly positive, and the constant $\delta$ is strictly positive $(\Delta H<0)$ for of exothermic reaction and strictly negative $(\Delta H>0)$ for the endothermic reactions. In this paper, we investigate the case of exothermic reaction (i.e., $T \geq T_{i n}$ ), or equivalently the case when $0 \leq x_{1}(z, t) \leq x_{1, \max }$.

This paper is organized as follows: The notations and preliminaries are given in Section 2. The existence of the global solution of the semi-linear state estimator of System (1)-(4) is proved in Section 3, and with additional assumption, we state the main result of the estimation error convergence. In Section 3, we present some simulation results. Finally, the main conclusions are outlined in Section 4. The background of our approach can be found in [7], [8] and [9].

\section{Notations and preliminaries}

Let $(X,\|\|)$ be a real Banach space, $(T(t))_{t \geq 0}$ is a $C_{0}$-semigroup of linear operators such that $\|T(t)\| \leq \exp (w t)$, for all $t \geq 0$, for some $w \in I R, A$ is the infinitesimal generator of $(T(t))_{t \geq 0}, N$ is a continuous function from a closed subset $D$ of $X$ into $X$ and $I$ is the identity operator on $X$. Recall that

$$
d(x ; D)=\inf \|x-y\|, \quad y \in D .
$$


For the following uncontrolled abstract Cauchy problem

$$
\left\{\begin{array}{l}
\dot{x}(t)=A x(t)+N(x(t)) \\
x(0)=x_{0} \in D
\end{array}\right.
$$

we sate this important theorem that ensure the existence of the global unique solution .

Theorem 2.1 ([7], p. 355) If the following conditions are satisfied:

i) $D$ is $(T(t))_{t \geq 0}$-invariant, i.e. $T(t) D \subset D$, for all $t \geq 0$;

ii) for all $x \in D$,

$$
\lim _{h \rightarrow 0^{+}} \frac{1}{h} d(x+h N(x) ; D)=0,
$$

iii) $N$ is continuous in $D$ and there exists $l_{N} \in I R^{+}$such that the operator $N-l_{N} I$ is dissipative on $D$ (i.e. $<\left(N-l_{N} I\right)(x-y), x-y>\leq 0, \quad \forall x, y \in D$.

Then, (9) has a unique mild solution $x\left(t, x_{0}\right)$ on $\left[0,+\infty\left[\right.\right.$, for all $x_{0} \in D$. Furthermore, if $(S(t))_{t \geq 0}$ is defined on $D$ by $S(t) x_{0}=x\left(t, x_{0}\right)$, for all $t \geq 0$ and $x_{0} \in D$, it is a nonlinear semigroup on $D$, with $(A+N)$ as its generator.

We state also the following Theorem that will be needed to prove the exponential convergence of the estimation error.

Theorem 2.2 ([9], p. 109) Let $\mathcal{A}$ be the infinitesimal generator of a $C_{0}$-semigroup $\left(T_{\mathcal{A}}(t)\right)_{t \geq 0}$ and $\mathcal{D}$ is linear bounded operator on $H$. The operator $\mathcal{A}+\mathcal{D}$ is the infinitesimal generator of a $C_{0}$-semigroup $\left(\bar{T}_{\mathcal{A}+\mathcal{D}}(t)\right)_{t \geq 0}$ which is the unique solution of the equation

$$
T_{\mathcal{A}+\mathcal{D}}(t) x_{0}=T(t) x_{0}+\int_{0}^{t} T(t-s) \mathcal{D} T_{\mathcal{A}+\mathcal{D}}(s) x_{0} d s, \forall x_{0} \in H .
$$

If in addition, $\|T(t)\| \leq M e^{\omega t}$, then

$$
\left\|T_{\mathcal{A}+\mathcal{D}}(t)\right\| \leq M e^{(\omega+M\|D\|) t}
$$

Throughout the sequel, we assume $H=L^{2}[0,1] \times L^{2}[0,1]$, the Hilbert space with the usual inner product

$$
<\left(x_{1}, x_{2}\right),\left(y_{1}, y_{2}\right)>=<x_{1}, y_{1}>_{L^{2}}+<x_{2}, y_{2}>_{L^{2}}
$$

and the induced norm

$$
\left\|\left(x_{1}, x_{2}\right)\right\|=\left(\left\|x_{1}\right\|_{L^{2}}^{2}+\left\|x_{2}\right\|_{L^{2}}^{2}\right)^{\frac{1}{2}}
$$

for all $\left(x_{1}, x_{2}\right)^{T}$ and $\left(y_{1}, y_{2}\right)^{T}$ in $H$.

Clearly the Hilbert space $H$ is a real Banach Lattice (for more details, see [10]) where, for all given $x=\left(x_{1}, x_{2}\right) \in H$, $y=\left(y_{1}, y_{2}\right) \in H$,

$x \leq y$ if and only if $x_{1} \leq y_{1}$ and $x_{2} \leq y_{2}$ for almost all $z \in[0,1]$.

And $H^{+}=\{x \in H: 0 \leq x\}$ is a positive cone. Let $\Gamma$ be a linear operator on $H$, then $\Gamma$ is said to be positive linear operator if $0 \leq \Gamma x$, for all $0 \leq x$, or equivalently $\Gamma H^{+} \subset H^{+}$.

As a useful criterion for the invariance condition given by (ii) of Theorem 2.1, we have the following lemma.

Lemma 2.3 [2] Let $T(t)$ be a strongly continuous semigroup of bounded linear operators on a real Banach lattice $X$, generated by $\mathcal{A}$, such that $\|T(t)\| \leq M \exp (w t)$ for all $t \geq 0$, for some $M \geq 1$ and $w \in I R$, then: $T(t)$ is positive if and only if the resolvent operator $R(\lambda, \mathcal{A}):=(\lambda I-\mathcal{A})^{-1}$ is a positive linear operator for all $\lambda>w$. 


\section{Semi-linear state estimator}

The (PDEs) (5)-(8) describing the exothermic reactor dynamics can be written on its compact form as

$$
\left\{\begin{array}{l}
\dot{x}(t)=A x(t)+N(x(t)) \\
x(0)=x_{0} \in D
\end{array}\right.
$$

where, $\mathrm{A}$ is the linear operator defined by:

$$
\begin{aligned}
D(A):=\{x & \left.=\left(\begin{array}{c}
x_{1} \\
x_{2}
\end{array}\right) \in H: x \text { absolutely continuous, } \frac{d x}{d z} \in H \text { and } x_{i=1,2}(0)=0\right\} \\
A\left(\begin{array}{l}
x_{1} \\
x_{2}
\end{array}\right): & :\left(\begin{array}{cc}
A_{1} & 0 \\
0 & A_{2}
\end{array}\right)\left(\begin{array}{c}
x_{1} \\
x_{2}
\end{array}\right) \\
& =\left(\begin{array}{cc}
-\frac{d .}{d z}-\beta I & 0 \\
0 & -\frac{d .}{d z}
\end{array}\right)\left(\begin{array}{l}
x_{1} \\
x_{2}
\end{array}\right) .
\end{aligned}
$$

It is shown in [1] that the linear operator $A$ is the infinitesimal generator of a $C_{0}$-semigroup of bounded linear operators on $H$, given by

$$
T_{A}(t)=\left(\begin{array}{cc}
T_{A_{1}}(t) & 0 \\
0 & T_{A_{2}}(t)
\end{array}\right)
$$

where $\left(T_{A_{1}}(t)\right.$ and $\left(T_{A_{2}}(t)\right.$ are the $C_{0}$-semigroups generated, respectively, by $A_{1}$ and $A_{2}$, such that for all $\left(x_{01}, x_{02}\right)^{T} \in$ $L^{2}(0,1) \times L^{2}(0,1)$, for all $(z, t) \in[0,1] \times I R$,

$$
\begin{aligned}
& \left(T_{A_{1}}(t) x_{01}\right)(z)= \begin{cases}\exp (-\beta t) x_{01}(z-t) & \text { if } z \geq t, \\
0 & \text { if } z<t,\end{cases} \\
& \left(T_{A_{2}}(t) x_{02}\right)(z)= \begin{cases}x_{02}(z-t) & \text { if } z \geq t, \\
0 & \text { if } z<t,\end{cases}
\end{aligned}
$$

Remark 3.1 i) It is easy to see from (13)-(14) that:

- For all $t \geq 0, T_{A}(t) H^{+} \subset H^{+}$, which is equivalent to $R(\lambda, A) H^{+} \subset H^{+}, \forall \lambda \in I R$ (by Lemma 2.3).

- And $\left\|T_{A}(t)\right\| \leq 1=\exp (w t)$, for all $t \geq 0$ (i.e. $w=0$ ).

ii) The $C_{0}$-semigroup $\left(T_{A}(t)\right)_{t \geq 0}$ is exponentially stable (see [1]), i.e. there exist constants $M, \rho$ in I $R^{*+}$ such that

$$
\left\|T_{A}(t)\right\| \leq M \exp (-\rho t), \forall t \geq 0
$$

In particular, there exists a time $\bar{t}$, such that

$$
\left\|T_{A}(t)\right\| \leq \exp (-\beta t), \forall t \geq \bar{t}
$$

The nonlinear operator $N$ is defined on

$$
D:=\left\{x=\left(\begin{array}{l}
x_{1} \\
x_{2}
\end{array}\right) \in H: 0 \leq x_{1}(z) \text { and } 0 \leq x_{2}(z) \leq 1 \text {, for almost all } z \in[0,1]\right\},
$$

for all $x=\left(\begin{array}{c}x_{1} \\ x_{2}\end{array}\right)$ in $\mathrm{D}$

$$
N(x)=\left(\begin{array}{c}
N_{1}(x) \\
N_{2}(x)
\end{array}\right):=\left(\begin{array}{c}
\alpha \delta\left(1-x_{2}\right) \exp \left(\frac{\mu x_{1}}{1+x_{1}}\right) \\
\alpha\left(1-x_{2}\right) \exp \left(\frac{\mu x_{1}}{1+x_{1}}\right)
\end{array}\right)
$$




\subsection{State estimator conception}

Hereafter we consider measurements of the state vector $x(t)$ are available at the reactor output only. In this case, the output function $y($.$) is defined as follows: we consider a (very small) finite interval with window width w$ at the reactor output $[1-w, 1]$ :

$$
\begin{aligned}
y(t) & =(C x)(t) \\
& :=\int_{0}^{1} \mathcal{X}_{[1-w, 1]}(a) x(a, t) d a, \forall t \in I R^{+}
\end{aligned}
$$

where, $\mathcal{X}_{[1-w, 1]}(a)=1$, if $a \in[1-w, 1]$ and $\mathcal{X}_{[1-w, 1]}(a)=0$, elsewhere

The observer operator $C: H \rightarrow I R^{2}$ is linear bounded and for all $x, y \in H \times I R^{2}$,

$$
\begin{aligned}
<C x, y>_{I R^{2}} & =<\int_{0}^{1} \mathcal{X}_{[1-w, 1]}(a) x(a, .) d a, y>_{I R^{2}} \\
& =\int_{0}^{1}<x(a, .), \mathcal{X}_{[1-w, 1]}(a) y>_{I R^{2}} d a
\end{aligned}
$$

The adjoint operator $C^{*}$ of $C$ is then defined for all $(z, t) \in[0,1] \times I R^{+}$by:

$$
\left(C^{*} y\right)(z)=\mathcal{X}_{[1-w, 1]}(z) y
$$

It is easy to see that for all $x \in H$,

$$
\left\|C^{*} C x\right\|^{2} \leq w\left\|\mathcal{X}_{[1-w, 1]}\right\|^{2}\|x\|^{2}
$$

what implies,

$$
\left\|C^{*} C\right\| \leq w .
$$

An observer design for the system (5)-(8), when only the temperature state is available for measurement, at the given reactor outlet, is given by

$$
\begin{array}{cc}
\frac{\partial \hat{x}_{1}}{\partial t}= & -\frac{\partial \hat{x}_{1}}{\partial z}-\beta \hat{x}_{1}+\alpha \delta\left(1-\hat{x}_{2}\right) \exp \left(\frac{\mu \hat{x}_{1}}{1+\hat{x}_{1}}\right)+g C_{1}^{*}\left(C_{1} x_{1}-C_{1} \hat{x}_{1}\right) \\
\frac{\partial \hat{x}_{2}}{\partial t}= & -\frac{\partial \hat{x}_{2}}{\partial z}+\alpha\left(1-\hat{x}_{2}\right) \exp \left(\frac{\mu \hat{x}_{1}}{1+\hat{x}_{1}}\right)
\end{array}
$$

with the boundary conditions:

$$
\hat{x}_{1}(z=0, t)=0, \hat{x}_{2}(z=0, t)=0
$$

and the initial conditions:

$$
\hat{x}_{1}(z, t=0)=\hat{x}_{1}^{0}, \hat{x}_{2}(z, t=0)=\hat{x}_{2}^{0}
$$

The system (17)-(20) can be written on its compact form as

$$
\left\{\begin{array}{l}
\dot{\hat{x}}(t)=\left(A-G C^{*} C\right) \hat{x}(t)+N(\hat{x}(t))+G C^{*} C x(t) \\
\hat{x}(0)=\hat{x}_{0} \in D
\end{array}\right.
$$

where, $x(t)=\left(x_{1}(., t), x_{2}(., t)\right)^{T}$ is the state variable of $(10)$ and $\hat{x}(t)=\left(\hat{x}_{1}(., t), \hat{x}_{2}(., t)\right)^{T}$. The linear operator $G$ satisfy: $G:=\left(\begin{array}{cc}G_{1} & 0 \\ 0 & 0\end{array}\right)=g I$, with $g$ is real number and $I$ is the identity operator of the Hilbert $H$.

The initial state $\left(x_{1}(0), x_{2}(0)\right)^{T}$ of $(8)$ is unknown while the initial state $\left(\hat{x}_{1}(0), \hat{x}_{2}(0)\right)^{T}$ of the observer can be assigned arbitrarily. Thus, the error between $\left(x_{1}(0), x_{2}(0)\right)^{T}$ and $\left(\hat{x}_{1}(0), \hat{x}_{2}(0)\right)^{T}$ is still an unknown quantity even if we know $\left(\hat{x}_{1}(0), \hat{x}_{2}(0)\right)^{T}$.

\subsection{Existence of the global solution}

Let consider the following coupled system, given for all $(x(0), \hat{x}(0))^{T} \in D \times D$, by

$$
\left(\begin{array}{c}
\dot{x}(t) \\
\dot{\hat{x}}(t)
\end{array}\right)=\left(\begin{array}{cc}
A & 0 \\
G C^{*} C & A-G C^{*} C
\end{array}\right)\left(\begin{array}{c}
\dot{x}(t) \\
\dot{\hat{x}}(t)
\end{array}\right)+\left(\begin{array}{c}
N(x(t)) \\
N(\hat{x}(t))
\end{array}\right)
$$

In order to investigate the asymptotic behavior of the estimation error $x()-.\hat{x}($.$) , we need to prove the existence$ of the solution of the augmented system (22), which remains in $D \times D$, on the whole interval $[0,+\infty)$, by applying Theorem 2.1. For this end we state the following lemmas concerning the nonlinearity involved in the dynamics (22). The proofs are similar to that given in [2]. 
Lemma 3.2 Consider the nonlinear operator (15). Then there exist $l_{N} \in I R^{+}$such that the operator $\left(\begin{array}{c}N-l_{N} I \\ N-l_{N} I\end{array}\right)$ is dissipative on $D \times D$.

Where $l_{N}:=\alpha \exp (\mu)(1+\mu)(1+|\delta|)$ with $\mu \exp (\mu)$ is a Lipschitz constant of the function $\exp \left(\frac{\mu s}{1+s}\right)$ on $[0,+\infty)$. Let define on $H \times H$, the distance

$$
d_{0}\left(\left(\begin{array}{l}
x \\
\hat{x}
\end{array}\right), D \times D\right)=\inf _{(y, \hat{y})^{T} \in D \times D} d\left(\left(\begin{array}{l}
x \\
\hat{x}
\end{array}\right),\left(\begin{array}{l}
y \\
\hat{y}
\end{array}\right)\right)
$$

Lemma 3.3 For all $(x, \hat{x})^{T} \in D \times D$,

$$
\lim _{h \rightarrow 0^{+}} \frac{1}{h} d_{0}\left(\left(\begin{array}{c}
x \\
\hat{x}
\end{array}\right)+h\left(\begin{array}{c}
N(x) \\
N(\hat{x})
\end{array}\right), D \times D\right)=0
$$

From the Theorem 2.2, the operator $A-G C^{*} C$ is the infinitesimal generator of a $C_{0}$-semigroup $\left(T_{A-G C^{*} C}(t)\right)_{t \geq 0}(:=$ $\left.\left(\left(\begin{array}{cc}T_{A_{1}-G_{1} C_{1}^{*} C_{1}}(t) & 0 \\ 0 & T_{A_{2}}(t)\end{array}\right)\right)\right)_{t \geq 0}$, where $\left(T_{A_{1}-G_{1} C_{1}^{*} C_{1}}(t)\right)_{t \geq 0}$ is the $C_{0}$-semigroup generated by the operator $A_{1}-$ $G_{1} C_{1}^{*} C_{1}$.

In order to study the invariance condition (i) of Theorem 2.1, it is useful to have the following Lemma:

Lemma 3.4 The $C_{0}$-semigroup $\left(T_{A-G C^{*} C}(t)\right)_{t \geq 0}$, is $D_{1} \times D_{2}$ invariant.

Proof 3.5 From Remark 3.1, we have $\left\|T_{A_{1}}(t)\right\| \leq \exp (\omega t)$ for all $t \geq 0$ with $\omega=0$, it follows by Theorem 2.2 that,

$$
\left\|T_{A_{1}-G_{1} C_{1}^{*} C_{1}}(t)\right\| \leq \exp \left(\left(\omega+\left\|G_{1} C_{1}^{*} C_{1}\right\|\right) t\right), \quad \forall t \geq 0 .
$$

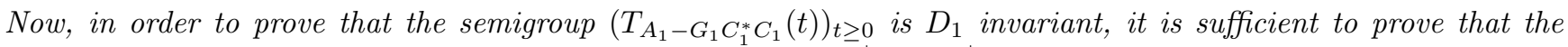
operator $A_{1}-G_{1} C_{1}^{*} C_{1}$ is positif (i.e., $R\left(\lambda, A_{1}-G_{1} C_{1}^{*} C_{1}\right) H^{+} \subset H^{+}$, for all $\lambda>\left\|G_{1} C_{1}^{*} C_{1}\right\|$, according the Proposition 2.3).

Consider that $\lambda>\left\|G_{1} C_{1}^{*} C_{1}\right\|$ and $(x, y) \in H^{+} \times D\left(A_{1}\right)$, such that:

$$
\left.R\left(\lambda, A_{1}-G_{1} C_{1}^{*} C_{1}\right) x=y \quad \text { (i.e., } \quad\left(\lambda I-A_{1}+G_{1} C_{1}^{*} C_{1}\right)^{-1} x=y\right)
$$

Let prove that $y \in H^{+}$. We have,

$$
x=\left(\lambda I-A_{1}\right) y+G_{1} C_{1}^{*} C_{1} y,
$$

whence,

$$
\left(\lambda I-A_{1}\right)^{-1} x=y+\left(\lambda I-A_{1}\right)^{-1} G_{1} C_{1}^{*} C_{1} y,
$$

then, for almost all $z \in[0,1]$,

$$
y(z)=\left(\left(\lambda I-A_{1}\right)^{-1} x\right)(z)-g\left(\int_{1-w}^{1} y(a) d a\right)\left(\left(\lambda I-A_{1}\right)^{-1} \mathcal{X}_{[1-w, 1]}(.)\right)(z),
$$

it follows that,

$$
y(z)=\left(R\left(\lambda, A_{1}\right) x\right)(z)-g\left(\int_{1-w}^{1} y(a) d a\right) \int_{0}^{z} \exp ^{-(\lambda+v) t} \mathcal{X}_{[1-w, 1]}(z-t) d t \mathcal{X}_{[1-w, 1]}(z) .
$$

From Remark 3.1, for all $\lambda \geq 0, R\left(\lambda, A_{1}\right) H^{+} \subseteq H^{+}$, whence,

$-y(z) \geq 0$ for almost all $z \in[0,1] \backslash[1-w, 1]$,

- and for almost all $z \in[1-w, 1]$, there is tree cases:

- if $\int_{1-w}^{1} y(a) d a=0$, thus $y(z) \geq 0$, for almost all $z \in[1-w, 1]$.

- if $\int_{1-w}^{1} y(a) d a<0$, thus $y(z) \geq 0$, for almost all $z \in[1-w, 1]$.

- if $\int_{1-w}^{1} y(a) d a>0$, suppose that there exist a subset non negligible $V \subseteq[1-w, 1]$ such that $y(z)<0, p p$. $z \in V$. We can suppose $[1-w, 1] \backslash V$ negligible in the size, since $w$ is very small number, it follows,

$$
\int_{1-w}^{1} y(a) d a\left(=\int_{V} y(a) d a+\int_{[1-w, 1] \backslash V} y(a) d a\right)<0
$$


what is contradictory. Thus, $y(z) \geq 0$ for almost all $z \in[0,1]$, and so $T_{A_{1}-G_{1} C_{1}^{*} C_{1}}(t) H^{+} \subset H^{+}$for all $t \geq 0$. It follows that, $T_{A_{1}-G_{1} C_{1}^{*} C_{1}}(t) D_{1} \subset D_{1}$. Besides, it is proved in [2] that $T_{A_{2}}(t) D_{2} \subset D_{2}$.

Therefore,

$$
T_{A-G C^{*} C}(t) D_{1} \times D_{2} \subset D_{1} \times D_{2}, \quad \forall t \geq 0
$$

The following proposition demonstrates the existence of the unique mild solution on $[0,+\infty)$ of the coupled nonlinear system (22):

Proposition 3.6 For all $\left(x_{0}, \hat{x}_{0}\right)^{T} \in D \times D$, the dynamic system (22) has a unique mild solution $\left(x\left(t, x_{0}\right), \hat{x}\left(t, \hat{x}_{0}\right)\right)^{T} \in$ $D \times D$, for all $t \geq 0$.

Proof 3.7 The linear bounded operator $\left(\begin{array}{cc}A & 0 \\ G C^{*} C & A-G C^{*} C\end{array}\right)$ is the generator of a $C_{0}$-semigroup defined for all $t \geq 0$ by

$$
\begin{aligned}
& T_{\mathcal{A}}(t)=\left(\begin{array}{cc}
T_{A}(t) & 0 \\
S(t) & T_{A-G C^{*} C}(t)
\end{array}\right) ; \\
& S(t)\left(\begin{array}{c}
x_{1} \\
x_{2}
\end{array}\right)=\left(\begin{array}{cc}
\int_{0}^{t} T_{A_{1}-G_{1} C_{1}^{*} C_{1}}(t-s) G_{1} C_{1}^{*} C_{1} T_{A_{1}}(s) x_{1} d s & 0 \\
0 & 0
\end{array}\right) .
\end{aligned}
$$

(see ([9], p. 30) for more details). In particular the $C_{0}$-semigroup $\left(T_{\mathcal{A}}(t)\right)_{t \geq 0}$ satisfy,

$$
T_{\mathcal{A}}(t) D \times D \subset D \times D, \text { for all } t \geq 0
$$

In deed, it is proved in [2] that $T_{A}(t)\left(=\left(\begin{array}{cc}T_{A_{2}}(.) & 0 \\ 0 & T_{A_{2}}(.)\end{array}\right)\right) D_{1} \times D_{2} \subseteq D_{1} \times D_{2}$.

Besides, from Lemma 3.4, for all $x\left(=\left(x_{1}, x_{2}\right)^{T}, \hat{x}\left(=\left(\hat{x}_{1}, \hat{x}_{2}\right)^{T}\right.\right.$ in $D$, and for all $t \geq 0$, we have

$$
T_{A-G C^{*} C}(t) \hat{x}=\left(\begin{array}{cc}
T_{A_{1}-G_{1} C_{1}^{*} C_{1}}(t) & 0 \\
0 & T_{A_{2}}(t)
\end{array}\right)\left(\begin{array}{c}
\hat{x}_{1} \\
\hat{x}_{2}
\end{array}\right) \in D_{1} \times D_{2}
$$

Since $0 \leq T_{A_{1}-G_{1} C_{1}^{*} C_{1}}(.) G_{1} C_{1}^{*} C_{1} T_{A_{1}}(.) x_{1}$, for all $x_{1} \in D_{1}$.

Then,

$$
S(t) x \in D, \quad \forall x \in D
$$

Hence, for all $t \geq 0$,

$$
\left(\begin{array}{cc}
\int_{0}^{t} T_{A_{1}-G_{1} C_{1}^{*} C_{1}}(t-s) G_{1} C_{1}^{*} C_{1} T_{A_{1}}(s) x_{1} d s+T_{A_{1}-G_{1} C_{1}^{*} C_{1}}(t) \hat{x}_{1} & 0 \\
0 & T_{A_{2}}(t) \hat{x}_{2}
\end{array}\right) \in D_{1} \times D_{2}
$$

It follows that,

$$
S(t) x+T_{A-L C}(t) \hat{x} \in D, \quad \forall t \geq 0
$$

Therefore, for all $(x, \hat{x})^{T} \in D \times D$,

$$
T_{\mathcal{A}}(t)\left(\begin{array}{c}
x \\
\hat{x}
\end{array}\right) \in D \times D, \quad \forall t \geq 0
$$

The condition (i) of Theorem 2.1 is thus satisfied. Conditions (ii) and (iii) of Theorem 2.1 follows respectively by Lemma 3.2 and Lemma 3.3. Finally by applying Theorem 2.1, the augmented system (22) admits a unique mild solution $(x, \hat{x})^{T} \in D \times D$ on the whole interval $[0,+\infty)$. 


\subsection{Convergence of the estimation error}

Now, we are ready to state the main result of this section,

Proposition 3.8 : Given the Plug Flow Reactor model (5)-(8). Suppose that there exists a bounded linear operator $G=g\left(\begin{array}{cc}I_{1} & 0 \\ 0 & 0\end{array}\right)$ with $g$ is a positif number, such that $g<\frac{\beta-l_{N}}{\omega}$, then the dynamic system (17)-(20) is an exponential observer for the system (5)-(8).

Proof 3.9 From Lemmas 3.2,3.3 and 3.4, we prove by applying Theorem 2.1 that the evolution of the estimation error, given by

$$
\dot{x}(t)-\dot{\hat{x}}(t)=\left(A-G C^{*} C\right)(x(t)-\hat{x}(t))+N(x(t))-N(\hat{x}(t)),
$$

admit for all $(x(0), \hat{x}(0))^{T} \in D \times D$ an unique mild solution on the whole interval $[0,+\infty)$, satisfying

$$
\begin{aligned}
x(t)-\hat{x}(t) & =T_{A-G C^{*} C}(t)(x(0)-\hat{x}(0)) \\
& +\int_{0}^{t} T_{A-G C^{*} C}(t-s)(N(x(s))-N(\hat{x}(s))) d s,
\end{aligned}
$$

such that, $(x(t), \hat{x}(t))^{T} \in D \times D$, for all $t \geq 0$.

It follows, for all $t \geq 0$, that

$$
\begin{aligned}
\|x(t)-\hat{x}(t)\| & \leq\left\|T_{A-G C^{*} C}(t)\right\|\|x(0)-\hat{x}(0)\| \\
& +\int_{0}^{t}\left\|T_{A-G C^{*} C}(t-s)\right\|\|N(x(s))-N(\hat{x}(s))\| d s,
\end{aligned}
$$

From Remark 3.1, there exists a time $\bar{t}$ such that $\left\|T_{A}(t)\right\| \leq \exp (-\beta t)$ for all $t \geq \bar{t}$. Consider $\alpha=\beta-g \omega$. It follows from Theorem [8],

$$
\left\|T_{A-G C^{*} C}(t)\right\| \leq \exp (-\alpha t), \quad \forall t \geq \bar{t}
$$

thus, for all $t \geq \bar{t}$,

$$
\|\exp (\alpha t)(x(t)-\hat{x}(t))\| \leq\|x(0)-\hat{x}(0)\|+l_{N} \int_{0}^{t}\|\exp (\alpha s)(x(s)-\hat{x}(s))\| d s,
$$

By applying Gronwall's Lemma ([9], p., 639),

$$
\|x(t)-\hat{x}(t)\| \leq M\|x(0)-\hat{x}(0)\| \exp \left(\left(-\alpha+l_{N}\right) t\right), \quad \forall t \geq \bar{t}
$$

\begin{tabular}{|c|c|c|}
\hline & & \\
\hline$v$ & 0.1 & $m \cdot s^{-1}$ \\
\hline $\mathrm{L}$ & 1 & $\mathrm{~m}$ \\
\hline$\delta$ & 0.25 & \\
\hline $\mathrm{E}$ & 11.250 & $\mathrm{cal} \cdot \mathrm{mol}^{-1}$ \\
\hline$k_{0}$ & $10^{6}$ & $s^{1}$ \\
\hline$\beta$ & 0.2 & $s^{1}$ \\
\hline$C_{i n}$ & 0.02 & $\mathrm{~mol} \cdot \mathrm{L}^{-1}$ \\
\hline $\mathrm{R}$ & 1.986 & $\mathrm{cal} \cdot \mathrm{mol}^{-1} \cdot L^{-1}$ \\
\hline$T_{i n}$ & 340 & $\mathrm{~K}$ \\
\hline
\end{tabular}

Therefore, the estimation error converges exponentially to zero if $g<\frac{\beta-l_{N}}{\omega}$.

\section{Simulation result}

In order to test the performance of the proposed observers, the equations have been integrated by using a backward finite difference approximation for the first-order space derivative $\left(\frac{\partial x}{\partial z} \simeq \frac{x\left(z_{i}, t\right)-x\left(z_{i-1}, t\right)}{\Delta z}\right)$, where $\Delta z$ is the spatial step (equal to 0.001), with the following set of parameter values (see [6], [5]):

Table 1: Process Parameters

The measurements are taken on the length interval $[3 * L / 4, L]$ i.e., $w=3 * L / 4$, and the process model has been arbitrary initialized with the constant profiles $x_{1}(0, z)=1, x_{2}(0, z)=0, \hat{x}_{1}(0, z)=0$, and $\hat{x}_{2}(0, z)=1$. In order to response to the assumption of the Propositions 3.8, we set $g=\frac{\beta-l_{N}}{\omega}$ for the observer design parameter.

Figure 1 shows respectively the time evolution of the temperature and concentration errors $x_{1}-\hat{x}_{1}$ and $x_{2}-\hat{x}_{2}$ related to the exponential reduced-order observer (17)-(20). 

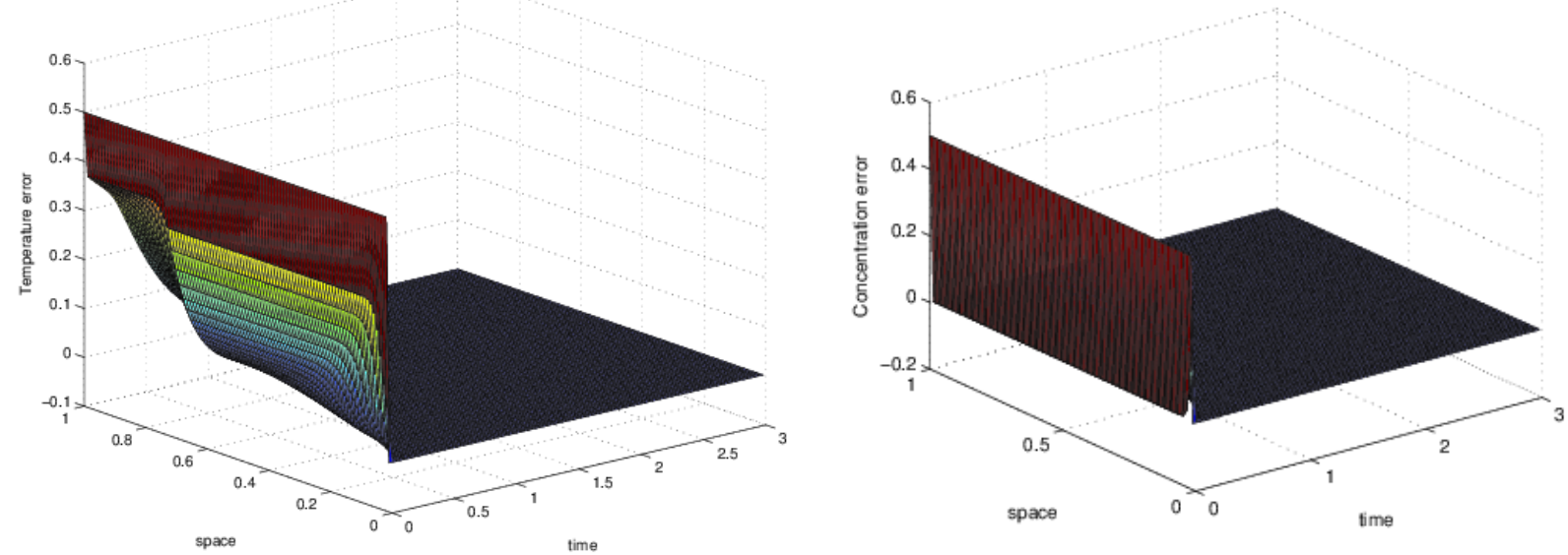

Figure 1: Evolution in time and space of the error on temperature in the left plot and of the error on reactant concentration in the right.

\section{Conclusions and prospects}

In this paper we present an exponential "Reduced-Order" observer to estimate the state variables initially unknown of a class of tubular reactor nonlinear models, namely exothermal Plug-Flow reactors involving sequential reactions for which the kinetics depends on temperature and reactant concentration. The given observer is based on measurements of the temperature at the reactor output only, and performed by a simulation study in which the parameters can be tuned by the user to satisfy specific needs in terms of convergence rate. It is shown in the simulations that the observer design is effective and satisfactory since it answers to difficulties of the reactant concentration measurements for a wide range of (bio)-chemical reactors.

\section{Acknowledgements}

This paper presents research results of the Moroccan "Programme Thématique d'Appui à la Recherche Scientifique" PROTARS III, initiated by the Moroccan "Centre National de la Recherche Scientifique et Technique" (CNRST). The scientific responsability rest with its authors. The work is also supported by the Belgian Programme on Interuniversity Poles of Attraction (PAI).

\section{References}

[1] J. Winkin, D. Dochain, P. Ligarius, "Dynamical Analysis of Distributed Parameter Tubular Reactors", Automatica 36 (2000) 349-361.

[2] M., Laabissi, M. E., Achhab, J., Winkin, D., Dochain,, "Trajectory analysis of nonisothermal tubular reactor nonlinear models", Syst. Control Lett 42 (2001) 169-184.

[3] C. Antoniades, P. D. Christofides, "Studies on nonlinear dynamics and control of tubular reactor with recycle", Nonlinear Anal 47, (2001) 5933-5944.

[4] S. Renou, M. Perrier, D. Dochain, S. Gendron, "Solution of the convection-dispersion-reaction equation by a sequencing method", Comput. Chem. Eng 27 (2003) 615-629.

[5] Y. Orlov, D. Dochain, "Discontinuous Feedback Stabilisation of Minimum-Phase Semilinear Infinite-Dimensional Systems With Application to Chemical Tubular Reactor Models", IEEE Trans. Aut., Vol.47, (2002), pp.1293-1304.

[6] I.Y. Smets, D. Dochain, J.F. Van Impe, "Optimal Temperature Control of a Steady-State Exothermic Plug-Flow Reactor", AIChE Journal, Vol.48, No.2, (2002), pp.279-286.

[7] R.H, Martin, Nonlinear operators and differential equations in Banach spaces, John Wiley and Sons, (1976). 
[8] A. Pazy, Semigroups of linear operators and applications to partial differential equations, Springer-Verlag, New York, (1983).

[9] R. F. Curtain, J. Zwart, An Introduction to Infinite Dimentional Linear Systems Theory, Springer, New York. (1995).

[10] R., NAGEL, One-Parameter Semigroups of Positive Operators, Lecture Notes in Mathematics, vol. 1184, Springer, New York, (1986). 